\title{
Rompimiento de la dormancia de yemas de vid (Vitis vinifera L.) mediante aplicaciones de extracto de ajo (Allium sativum L.) bajo condiciones del trópico alto
}

\section{Breaking the dormancy of grapevine (Vitis vinifera L.) buds with the application of garlic extract (Allium sativum L.) under tropical high-altitude conditions}
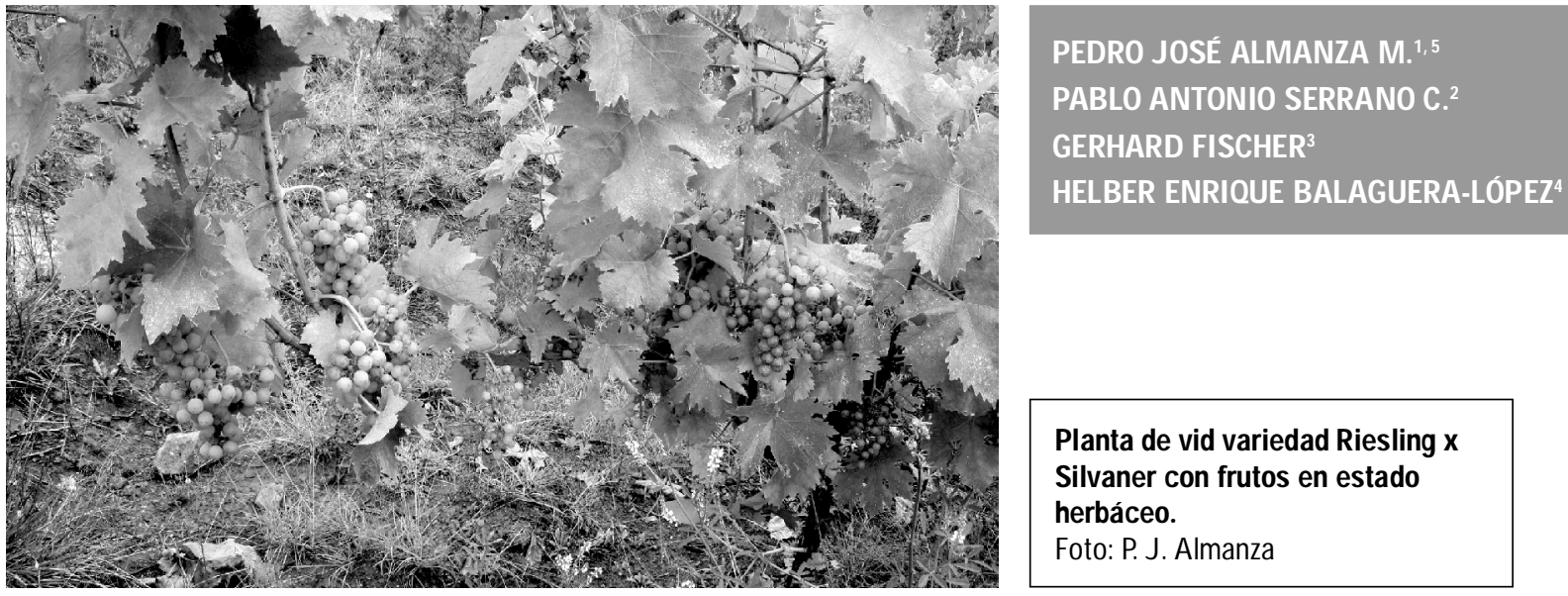

\section{RESUMEN}

La vid para elaborar vinos se ha cultivado durantelos últimos 25 años en 18 municipios deBoyacá (Colombia), donde las condiciones de trópico alto no presentan ambientes de frío suficiente, lo que genera una brotación baja e irregular, y, en consecuencia, producciones bajas y desuniformes. El ajo se ha reconocido como agente inductor de brotación, pero no se ha evaluado bajo condiciones tropicales. Se evaluó el efecto del extracto de ajo en la inducción dela brotación en uva dela variedad clonal Riesling x Silvaner, en el municipio deCorrales (Boyacá). Seempleó un diseño completamenteal azar con cuatro tratamientos (extracto deajo al $100 \%$, extracto de ajo al 50\%, cianamida hidrogenada y testigo) y cinco repeticiones. Los tratamientos fueron aplicados directamente sobre las yemas inmediatamente después de la poda, con ayuda de un pincel. Se determinó el porcentaje de brotación (PB), el tiempo medio debrotación (TM B), la velocidad media de brotación (VM B), el área foliar y el número de hojas, brotes y racimos. La aplicación de ajo al $50 \%$ presentó significativamente el mayor PB, el menor TM B y la mayor VM B; además, pero sin diferencias estadísticas, una respuesta favorable en el área foliar y en el número dehojas, brotes y racimos de plantas de uva. Se concluye quelas aplicaciones de ajo son una alternativa importanteen la inducción de la brotación en yemas de la variedad Riesling x Silvaner.

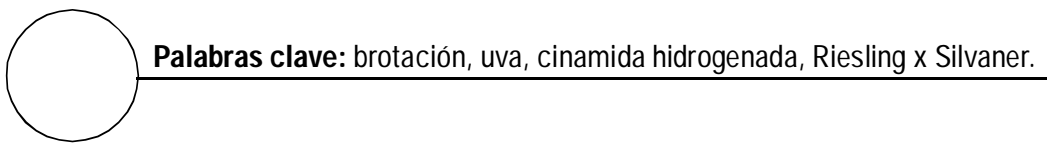

1 Facultad de Ciencias Agropecuarias, Grupo de Investigación Ecofisiología Vegetal, U niversi dad Pedagógica y Tecnológica de Colombia, Tunja (Colombia).

2 Facultad deCiencias Agropecuarias, Grupo de Investigación GIPSO, Universidad Pedagógica y Tecnológica de Colombia, Tunja (Colombia).

3 Facultad de Agronomía, Departamento de Agronomía, Universidad N acional deC olombia, Bogotá (Colombia).

4 Facultad de Agronomía, Programa de M aestría en Ciencias Agrarias con énfasis en Fisiología de Cultivos, Universidad Nacional de Colombia, Bogotá; Grupo de Investigaci ones Agrícolas, Facultad de Ciencias Agropecuarias, U niversidad Pedagógi ca y Tecnológica de Colombia, Tunja (Colombia).

5 Autor para correspondencia. ppcalma@gmail.com 


\section{ABSTRACT}

Grapes for winemaking have been growing over the past 25 years in 18 municipalities of Boyacá (Colombia). The climatic conditions of high elevations in tropical zones do not provide cold enough conditions, resulting in low and irregular bud breaking, and generating low and uneven yields. Garlic has been recognized as an inducer of bud breaking, but it has not been evaluated under tropical conditions. Therefore, this study evaluated the effect of garlic extract on the induction of breaking bud dormancy in the clonal grape variety Riesling $x$ Silvaner in the municipality of Corrales (Boyacá). A completely randomized design was used with four treatments (garlic extract 100\%, garlic extract 50\%, hydrogen cyanamide and control) with five repetitions. Treatments were applied directly on the buds immediately after pruning, using a brush. The percentage of bud breaking (PBB), average time of bud breaking (ATB), average speed of bud breaking (ASB), leaf area, and number of leaves, shoots and panicles were determined. With statistical differences, the application of garlic $50 \%$ had significantly thehighest PBB, thelow est AT B and thehigher ASB, and, without statistical differences, favorable responses w ere found in leaf area, number of leaves, shoots and trusses of grape plants. It can be concluded that the application of garlic is an important alternative in the induction of bud breaking in the Riesling x Silvaner variety.

Keyw ords: bud breaking, wine, hydrogen cyanamid, Riesling x Silvaner.

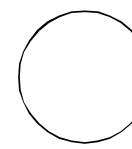

La producción mundial de uva en el 2009 fue de 66.935.199 t, cultivadas en 7.437.141 ha, de las cuales Colombia tenía sembradas 2.581 ha (FAO, 2010); en el país, bajo condiciones de clima frío tropical, los principales productores de vid para producción de vino se encuentran en el departamento de Boyacá, en 16 municipios de las provincias de Sugamuxi, Valderrama, Tundama, Centro, N orte y Gutiérrez, y en dos de la zona de Ricaurte Alto (Gómez, 2004), en altitudes entre 2.200 y $2.560 \mathrm{msnm}$, donde se ha cultivado durantelos últimos 24 años (Q uijano, 2004).

En la zona templada, la vid produceuva una vez al año; durante el invierno las plantas entran en dormancia; en condiciones tropicales se pueden obtener hasta 2,5 cosechas al año, gracias al manejo y a las condiciones agroclimáticas (Quijano, 2001). Las plantas de uva requieren de acumulación de un determinado número de horas frío para salir del periodo de endodormancia; según Westwood (1993), este valor depende de la variedad (150 a $1.200 \mathrm{~h}$ );
Dokoozlian (1999) menciona que requieren de 50 a 400 horas frío. La acumulación de horas frío se da bajo temperaturas inferiores a 7으 (M artínez, 1991). La falta de acumulación de horas frío produce brotación desuniforme y retrazo en la maduración de frutos (Pinto et al., 2003); por tanto, en zonas tropicales es necesaria la aplicación de compensadores químicos de frío.

La vid presenta yemas mixtas (reproductivas y vegetativas), que se pueden clasificar según el momento de brotación en: yemas prontas o de brotación anticipada, yemas latentes, yemas adventicias, yemas axilares y yemas basilares 0 ciegas (Reynier, 1995); están constituidas exteriormente por escamas protectoras de forma triangular y decolor pardo, bajo las cual es existe una segunda capa protectora llamada al godón o borra, de color blanquecino; ambas estructuras protegen los meristemos terminales o ápices vegetativos del frío. La diferenciación de las yemas consiste en la organización de los meristemos y esbozos de hojas, racimos y zarcillos para que en el siguiente ciclo se origine 
el nuevo brote cargador; terminada la diferenciación de cada yema se inicia la etapa de paradormancia, durante la cual la mayoría de yemas basales tienen la capacidad de brotar, pero permanecen en reposo debido a la influencia ejercida por las yemas apicales y anticipadas de los sarmientos que están aún en crecimiento (Pinto et al., 2003); esta capacidad de brotación se va perdiendo a medida que se lignifica el sarmiento, por la acumulación de lignina. Este periodo es conocido como agostamiento, y coincide con la terminación del crecimiento del sarmiento e inicio de la endodormancia de las yemas. Durante este periodo las yemas pierden en dos a tres semanas su capacidad de brotación. D e este estado solo salen las yemas una vez han cumplido el requerimiento de acumulación de horas frío quele permita pasar a la ecodormancia, permaneciendo en este estado hasta que las condiciones de temperatura aseguren el desarrollo del nuevo pámpano (Reynier, 1995).

Bajo condiciones de climas tropicales, como el caso de Colombia, en donde la vid no alcanza a acumular el número dehoras frío necesarias para brotar, es básica la aplicación de reguladores fisiológicos que influyen en la brotación y en la acumulación de horas frío, como, por ejemplo, cianamida hidrogenada $\left(\mathrm{H}_{2} \mathrm{CN}_{2}\right)$, producto que actualmente es el más utilizado en viticultura en todo el mundo; en 2001, aproximadamente $112.490 \mathrm{~kg}$ de cianamida de hidrógeno fueron utilizados en Estados Unidos, y $36.287 \mathrm{~kg}$, en Italia (Settimi et al., 2005). Las aplicaciones se realizan en forma de aspersión, o con pincel o rodillo, sobre las yemas, en dosis que van entre 35 a $50 \mathrm{~cm}^{3} \mathrm{~L}^{-1}$ de agua; es importante que esta labor, para que surta efecto, se realice en un tiempo máximo de $48 \mathrm{~h}$ después de poda, en días soleados y con suelos húmedos. Si no se aplica el compensador, la brotación no es homogénea y se provocan ciclos fenológicos disparejos, que disminuyen la calidad del fruto, debido a que los racimos maduran escalonada y desuniformemente (Toro, 1996).

Buscando nuevas alternativas a la ruptura de la dormancia, Kubota y Miyamuki (1992) encontraron que la pasta de ajo aplicada a la superficie de las estacas de vid Moscatel de
Alejandría, poco después de la poda, es más eficiente que la cianamida cálcica $\left(\mathrm{CaCN}_{2}\right)$, el producto más utilizado para vides en Japón. También fueron satisfactorios los resultados obtenidos con aceite deajo al $20 \%$ en vides Pione y Thompson Seedless (Kubota et al., 2000). Botel ho et al. (2009) encontraron brotación en un $37 \%$ y $75 \%$ en esquejes de vid cv. Cabernet Sauvignon, tratados con extracto de ajo al 3\%, sometido a 0 y 168 horas frío $\left(<7,0^{\circ} \mathrm{C}\right)$ respectivamente. Según Kubota et al. (2003) y Kubota et al. (1999), la sustancia activa en el extracto de ajo responsable de la pérdida de dormancia comprendecompuestos volátiles que contienen azufre y un grupo de alilo $\left(\mathrm{CH}_{2} \mathrm{CHCH}_{2}\right)$.

Vargas-A rispuro et al. (2008) aislaron y evaluaron diferentes compuestos derivados de ajo para medir su efecto en la inducción de brotación de yemas en estacas de un cultivo orgánico de uva de mesa (cultivares Flame Seedless y Perlette). D entro de los compuestos aislados eidentificados químicamente, se encuentran: allicina, dialil disulfuro, dialil trisulfuro, 3-vinil-(4H )-1,2-ditiin y 2-vinil-(3H)- 1,3-ditiin, S-metilcisteina sulfóxido, dimetil disulfuro, dimetil trisulfuro y dimetil tiosulfonato. Se encontró que todos los compuestos promovieron la brotación de las yemas, y que los más efectivos fueron los compuestos volátiles de S-metilcisteina sulfóxido; es así como los compuestos derivados de azufre pueden inducir brotación en las yemas (Botelho et al., 2007).

Teniendo en cuenta la reducción o eliminación del uso de sustancias sintéticas que se emplean en los sistemas de producción sostenibles en frutales, y la búsqueda de nuevas alternativas para la salida de dormancia de los frutales en zonas tropicales, el extracto de ajo puede convertirse en una alternativa muy importante en la viticultura regional, ya que la cianamida hidrogenada es un producto que está clasificado por la Agencia deProtección M edioambiental de los Estados Unidos en la categoría más el evada de toxicidad (categoría I); además, el registro de ese producto es objeto de evaluación por parte de la Unión Europea (Settimi et al., 2005). Por tanto, el objetivo de la investigación fueevaluar 
el efecto del extracto de ajo (Allium sativum L.) como inductor de la brotación en plantas de vid de la variedad clonal Riesling $x$ Silvaner, en el municipio de Corrales (Boyacá).

\section{MATERIALES Y MÉTODOS}

La investigación se realizó durante los meses de abril y mayo de 2009 en el viñedo del municipio deC orrales, Boyacá, situado a 548'30" latitud N y $72^{\circ} 58^{\prime} 35^{\prime \prime}$ longitud W, a una altitud de 2.450 msnm; el clima de la zona se caracteriza por presentar una temperatura promedio anual de $16,5^{\circ} \mathrm{C}$ y precipitación bimodal media de $750 \mathrm{~mm}$ año-1 , con dos picos ubicados entre abril-mayo y octubre-noviembre; existe escasa nubosidad y alta insolación, que alcanza un promedio de 476 cal cm $\mathrm{cm}^{-2} \mathrm{~d}^{-1}$, en $6,5 \mathrm{~h} \mathrm{~d}^{-1}$; los suelos son pedregosos de textura liviana, del tipo franco-arenoso y fertilidad natural baja (EOT Corrales, 2009).

En el momento de la evaluación, las plantas tenían una edad de 8 años y corresponden a la selección clonal de Vitis vinifera $L$. variedad Riesling $x$ Silvaner $(\mathrm{R} \times \mathrm{S})$, originarias deAlemania y procedentes del viñedo y cava «Loma de Puntalarga», en N obsa (Boyacá). Las plantas están sembradas a distancia de $1,2 \mathrm{~m} \times 0,8 \mathrm{~m}$. El sistema de manejo es el de tipo Guyot simple con dos pulgares y conducción en espaldera a tres alambres.

Se empleó un diseño completamenteal azar con cuatro tratamientos correspondientes a inductores debrotación (ajo concentrado [100\%], ajo al $50 \%$, un testigo absoluto [agua destilada] y cianamida hidrogenada como testigo comercial [Dormex ${ }^{\circledR}$ al $\left.5 \%\right]$ ), con cinco repeticiones, para un total de 20 unidades experimentales. Cada unidad estuvo compuesta por 20 plantas. Los tratamientos fueron aplicados una sola vez y directamente sobre las yemas, hasta lograr una cobertura total con ayuda de un pincel. Para el tratamiento de ajo al $100 \%$, los dientes de ajo fueron macerados en un mortero de porcelana, mientras que en el tratamiento del $50 \%$ el ajo fue licuado y diluido en agua en relación $1: 1 \mathrm{~m} / \mathrm{v}$.
Desdeel momento en que brotaron las primeras yemas y hasta el final dela brotación sehicieron lecturas cada $3 d$, con el fin deregistrar el número de yemas brotadas; con estos datos y con las fórmulas de la tabla 1 secalculó el porcentaje de brotación (PB), tiempo medio de brotación (TM B) y velocidad media de brotación (VM B). Seconsideraron yemas brotadas cuando apareció la etapa C deBaggliolini o 05 de la BBCH (Salazar y M elgarejo, 2005), que consiste en la aparición de punta verde constituida por el brote joven, después de haberse activado el desarrollo de la yema en reposo. A los $50 \mathrm{~d}$ después de la poda (ddp) se determinó el área foliar « in situ»mediante medidor portátil $\mathrm{Cl}-202$ Seedmech, número de hojas, racimos y brotes de plantas deuva.

Para analizar el efecto de los compuestos sobre la brotación, se realizó un análisis de varianza (Anava); las medidas se compararon por la prueba de Tukey $(P<0,05)$, para lo cual se utilizó el software SAS v.8e.

\section{RESULTADOS Y DISCUSIÓN}

\section{Brotación}

La aplicación del extracto deajo (50\% y $100 \%)$ y la cianamida hidrogenada presentaron estadísticamente $(P<0,01)$ mayor brotación que el testigo. El mayor porcentaje de brotación se obtuvo con el extracto de ajo al $50 \%$ y la cianamida hidrogenada, con $96,6 \%$; el testigo apenas alcanzó el 83,3\% (figura 1). La aplicación de extracto de ajo al 50\% generó el menor T M B, con 45,25 d ( $P<0,01$; figura 2$)$, al igual que la mayor VM B (0,256 yemas/día), con diferencias estadísticas solamente respecto al testigo $(P<0,01$; figura 3$)$.

A los $26 \mathrm{ddp}$, los tratamientos de ajo al 50\% y al $100 \%$ respondieron con $13,3 \%$ y $6,6 \%$ deyemas, respectivamente, mientras que la aplicación de agua como testigo absoluto logró brotación a los 29 ddp con $11,6 \%$. En todos los casos, las primeras yemas en brotar fueron las insertadas sobre los pulgares, debido a la fuerte fertilidad existente en estas estructuras. De acuerdo con Valor y Bautista (1997), la fertilidad en V. vinifera tiende 


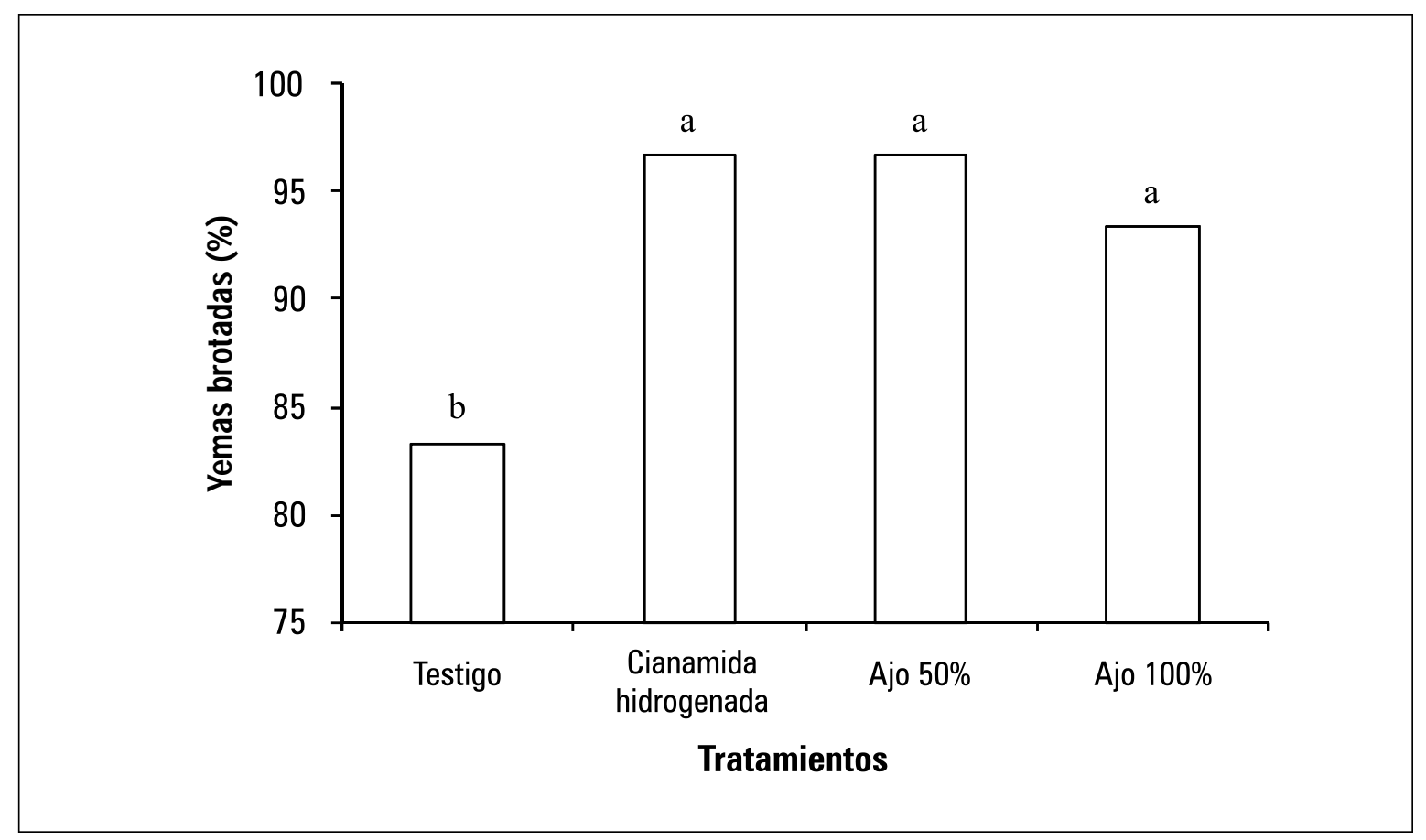

Figura 1. Efecto del extracto de ajo sobre el porcentaje de yemas brotadas de uva bajo condiciones del trópico alto. Promedios con letras distintas indican diferencia significtiva según la prueba de Tukey $(P<0,05)$.

a aumentar de la base hacia la porción media, donde se alcanza un máximo a partir del cual comienza a decrecer. Sin embargo, el patrón de fertilidad deyemas presenta muchas variaciones

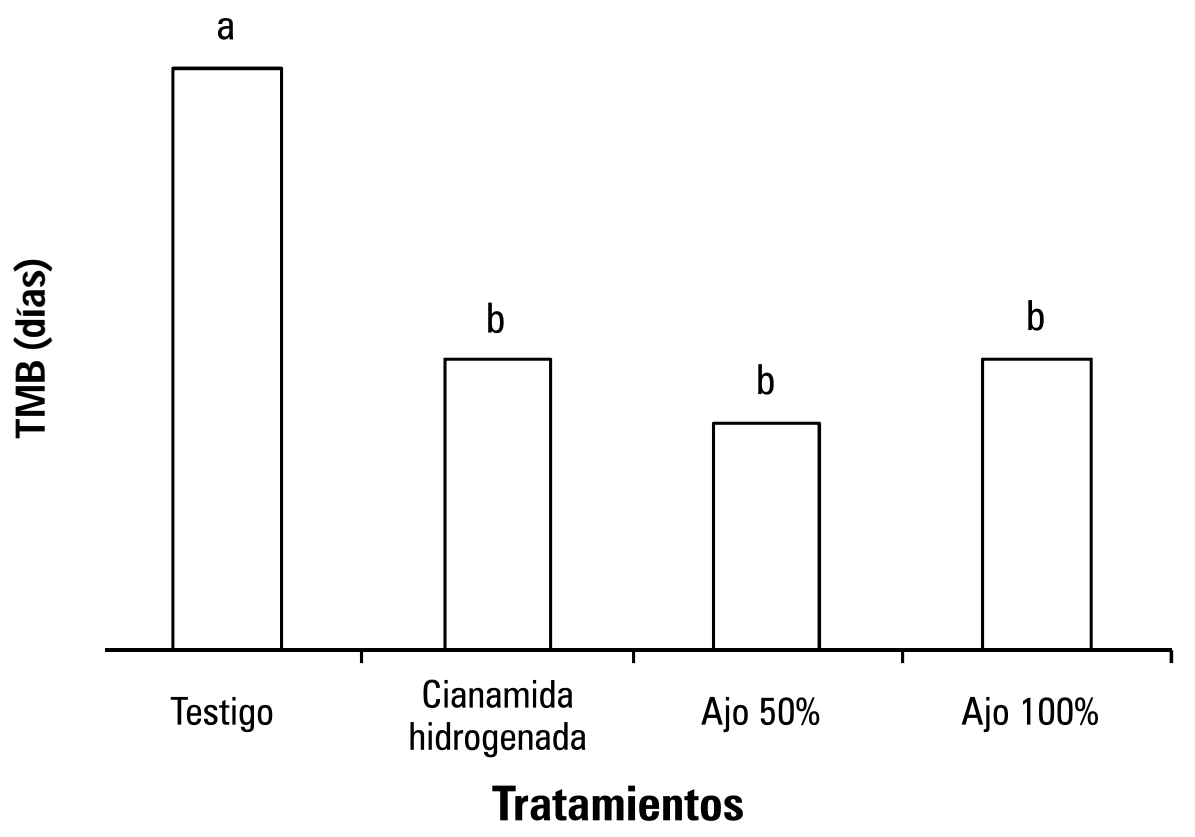

Figura 2. Efecto del extracto de ajo sobre el tiempo medio de brotación (TMB) de yemas de uva bajo condiciones del trópico alto. Promedios con letras distintas indican diferencia significtiva según la prueba de Tukey $(\mathrm{P}<0,05)$. 


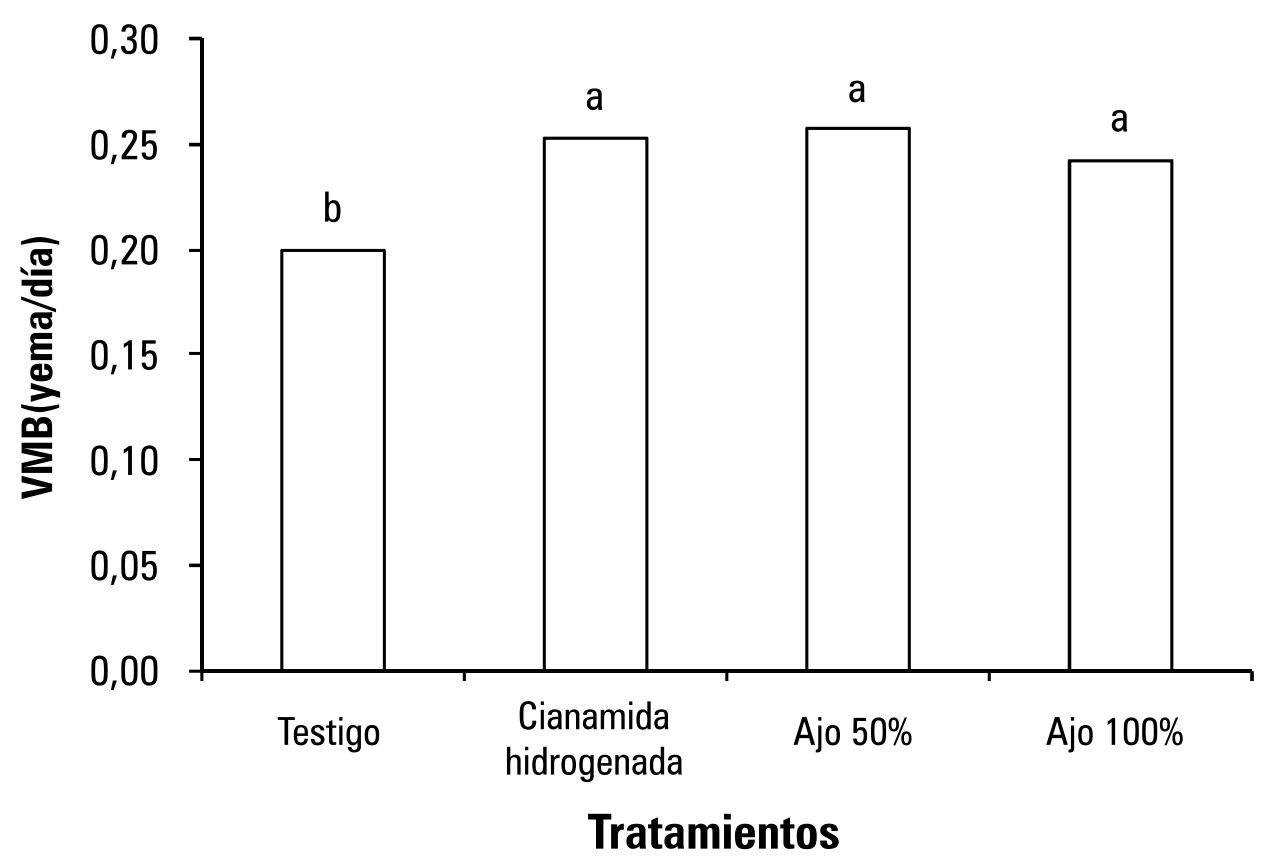

Figura 3. Efecto del extracto de ajo sobre la velocidad media de brotación (TM B) de yemas de uva bajo condiciones del trópico alto. Promedios con letras distintas indican diferencia significtiva según la prueba de Tukey $(P<0,05)$.

entre cultivares y entre diferentes ciclos de crecimiento de un mismo cultivar.

Según Butrosse(1974), en el proceso debrotación la temperatura juega un papel determinante. Las temperaturas del trópico y, posiblemente, la alta radiación UV-B hacen que la brotación no sea uniforme en las yemas más alejadas del tronco, trayendo consigo una baja uniformidad en la maduración, que afecta la calidad del fruto al momento de la cosecha (Val or y Bautista, 1997). Valor y Bautista (2001), evaluando la fenología decuatro cultivares de vid para vino, encontraron brotaciones entre9 y 10 ddp, y Vargas et al. (1994) mencionan que la duración de la brotación se hace mayor debido a las condiciones climáticas, especialmente a lo relacionado con la duración del fotoperiodo.

Estos resultados comprueban que el extracto de ajo es un inductor del rompimiento del estado de dormancia en cultivares de vid en zonas tropicales de altitud, en donde existe deficiencia en la acumulación de temperaturas inferiores a $7^{\circ} \mathrm{C}$, como es el caso del municipio de Corrales; sin embargo, Kubota et al. (2003) mencionan que a pesar de la eficacia de este compuesto, sus ef ectos duranteel tiempo y la concentración aún no se han investigado en relación con las fases de reposo vegetativo.

Kubota et al. (2000) determinaron que el disulfuro dealilo es la sustancia más importante encontrada en el ajo que inducebrotación en uva; este compuesto también ha sido eficiente en la ruptura de la dormancia de cormos, tubérculos (Hosoki et al., 1985) y en yemas de manzano (Wang y Faust, 1994).

Según Pinto et al. (2007), los principales mecanismos implicados en la ruptura de la dormancia de plantas frutales de clima templado, relacionados con la inducción de estrés oxidativo, es el peróxido de hidrógeno $\left(\mathrm{H}_{2} \mathrm{O}_{2}\right)$; este actúa como una señal química, provocando la activación de la expresión de genes que directa o indirectamente inducen cambios metabólicos detectados por otras moléculas, tales como kinasa, que activan o reprimen la expresión de los genes implicados en el rompimiento de la dormancia. Según Pérez y Lira (2005), la aplicación de cianamida hidrogenada, como la 
exposición al frío, inhibela actividad de catalasa, la principal enzima responsabledela degradación de peróxido de hidrógeno en la yema de la vid.

Las sustancias activas de ajo responsables de la sal ida de la dormancia en la uva son compuestos volátiles de sulfito de alilo, con dos grupos, en particular el dial disulfito; aunque el dial mono, el tri y el tetra sulfitos también pueden estar involucrados (Kubota et al., 2003); posiblemente, estos compuestos actúan por el mismo mecanismo propuesto por Pinto et al. (2007) para el rompimiento de dormancia de las plantas de frutas caducifolias, es decir, por el estrés oxidativo quese genera a través dela acumulación $\operatorname{deH}_{2} \mathrm{O}_{2}$.
Efectos favorables dela aplicación deajo sobrela brotación de diferentes variedades de uva también fueron encontrados por Kubota et al. $(1987,2000,2002)$ y Kubota y M iyamuki (1992), utilizando pasta de ajo, y por Kubota et al. (1999a) con ajo rallado hervido. Por su parte, Bothelo et al. (2007) reportan un efecto positivo con extracto de ajo en la brotación de manzana 'Royal Gala', respuesta que es directamente proporcional a la dosis ( $1 \%, 5 \%$ y $10 \%)$, resultado que difiere parcialmente de lo encontrado en la presente investigación, ya que el tratamiento de ajo al $50 \%$ fue levemente superior al de ajo al $100 \%$, lo cual se puede deber a que este último tratamiento, al ser más viscoso, no garantizó un recubrimiento uniforme de las yemas.

Tabla 1. Fórmulas para calcular los diferentes índices de brotación utilizados en el rompimiento de la dormancia de yemas de uva mediante aplicaciones de extracto de ajo, bajo condiciones del trópico alto.

\begin{tabular}{|l|c|c|}
\hline \multicolumn{1}{|c|}{ Variable } & Ecuación & Unidades \\
\hline Velocidad media de brotación & $\mathrm{VMB}=\sum\left(\frac{\mathrm{n}_{\mathrm{i}}}{\mathrm{t}_{\mathrm{i}}}\right)$ & yemas /día \\
\hline Tiempo medio de brotación & $\mathrm{TMB}=\frac{\mathrm{N} *\left(\mathrm{~A}_{1}+\mathrm{A}_{2}+\mathrm{A}_{\mathrm{X}}\right)}{\left(\mathrm{A}_{1} * \mathrm{~T}_{1}+\mathrm{A}_{2} * \mathrm{~T}_{2}+\mathrm{A}_{\mathrm{X}} * \mathrm{~T}_{\mathrm{X}}\right)}$ & días \\
\hline Porcentaje de brotación & $\mathrm{PB}=\left(\frac{\mathrm{N}}{\mathrm{Nt}}\right) * 100$ & $\%$ \\
\hline
\end{tabular}

$n_{i}$, Número de yemas brotadas en el i-ésimo día; $t$, tiempo en días para la brotación en el i-ésimo día; $N$, numero de yemas brotadas; $A_{1}, A_{2}, \ldots, A_{x^{\prime}}$ número de yemas brotadas en el día 1 , en el día 2 , y en el día $x_{;} T_{1}, T_{2}, \ldots, T_{x^{\prime}}$ número de días entre la aplicación de los tratamientos y el primer día 1 de brotación, entre el día 2 y entre el día $x$; Nt, número de yemas totales.

Tabla 2. Efecto del extracto de ajo sobre el área foliar y el número de hojas, racimos y brotes de plantas de uva, bajo condiciones del trópico alto.

\begin{tabular}{|l|c|c|c|c|}
\hline \multicolumn{1}{|c|}{ Tratamientos } & Área foliar $\left(\mathrm{cm}^{2}\right)$ & Número de hojas & Número de racimos & Número de brotes \\
\hline Testigo & $1.223,79 \mathrm{~b}$ & $43 \mathrm{a}$ & $10,4 \mathrm{a}$ & $28,2 \mathrm{a}$ \\
\hline Cianamida hidrogenada & $1.984,64 \mathrm{a}$ & $52 \mathrm{a}$ & $10,8 \mathrm{a}$ & $42,4 \mathrm{a}$ \\
\hline Ajo $50 \%$ & $1.926,59 \mathrm{a}$ & $58 \mathrm{a}$ & $13,4 \mathrm{a}$ & $37 \mathrm{a}$ \\
\hline Ajo $100 \%$ & $1.660,64 \mathrm{a}$ & $49 \mathrm{a}$ & $12,6 \mathrm{a}$ & $31,4 \mathrm{a}$ \\
\hline Significancia & $*$ & $\mathrm{~ns}$ & $\mathrm{~ns}$ & $\mathrm{~ns}$ \\
\hline
\end{tabular}

Promedios con letras distintas indican diferencia significtiva según la prueba de Tukey (P 0,05$)$. ns: no significante; * diferencias estadísticas al $5 \%$ de acuerdo con el Anava. 


\section{Área foliar, número de hojas, racimos y brotes}

La mayor respuesta del área foliar la presentó la aplicación de cianamida hidrogenada, con $1.984,64 \mathrm{~cm}^{2} /$ planta, seguida por el tratamiento deajo al $50 \%$, con $1.926,6 \mathrm{~cm}^{2} /$ planta; solo hubo diferencias significativas $(P<0,05)$ respecto al testigo, que generó la menor cantidad de área foliar (tabla 2). Los tratamientos de ajo y cianamida hidrogenada desplegaron la primera hoja a los $26 \mathrm{ddp}$. El mayor número de hojas se obtuvo con la aplicación de ajo al 50\%, sin embargo, no se presentaron diferencias estadísticas (tabla 1). En el número de brotes y de racimos tampoco se presentaron diferencias estadísticas; a pesar deesto, hubo mayor número de racimos con la aplicación de ajo al 50\%, y de brotes, con la cianamida hidrogenada; en los dos casos, el testigo mostró los valores más bajos (tabla 2).

La respuesta del crecimiento es dependiente del número de hojas y de la cantidad de área foliar expuesta a la luz. Las hojas son las directamente responsables de muchas funciones para el crecimiento y desarrollo de las plantas, como la intercepción y absorción de energía luminosa, la fotosíntesis, la respiración, la fotorrespiración y la traslocación de fotoasimilados hacia los sumideros (Disegna et al., 2005). D eacuerdo con $\mathrm{N}$ avarrete (2003), de la capacidad quetengan las plantas para realizar estos procesos metabólicos depende su estado de desarrollo. La deficiencia del aparato fotosintético implica un crecimiento y desarrollo lentos de la planta, pues implica menor tasa fotosintética y, por tanto, afecta el crecimiento de los pámpanos.

De acuerdo con lo anterior, las aplicaciones de ajo y de cianamida hidrogenada estarían favoreciendo de manera indirecta el crecimiento y desarrollo de la vid, pues aceleraron la brotación deyemas y la aparición de hojas, queiniciaron el proceso fotosintético más rápidamente, y aunque no hubo diferencias en el número de hojas, racimos y brotes, muy posiblementesí se podrá obtener una respuesta favorable y diferencial en el tamaño delas bayas y del racimo de las plantas tratadas con ajo y la cianamida; esto debido a que dichas plantas poseen mayor área foliar que les garantizará mayor producción de fotoasimilados que serán translocados en su mayoría a los órganos vertederos; asimismo, es probable que la calidad de las bayas y del vino sea mayor.

En concordancia, Kubota et al. (2000) evaluaron la aplicación deajo, disulfuro dialilo y cianamida hidrogenada en el rompimiento dela dormancia de plantas de uva crs. Pione y Thompson Seedless; encontraron que las plantas que brotaron primero presentaron brotes de mayor longitud en Thompson Seedless, y observaron mayor número de racimos con ajo y disulfuro dialilo, pero en el primer cultivar no se obtuvo efecto significativo en este parámetro. Estos autores comentan que el crecimiento vigoroso de los brotes, generado por una brotación más rápida, podría estar relacionado con el aborto de algunos racimos deflores, lo cual podría también explicar los resultados encontrados en el presente estudio.

\section{CONCLUSIONES}

La aplicación de ajo al 50\% presentó el mayor porcentaje de brotación, el menor tiempo medio de brotación, la mayor velocidad media de brotación y una respuesta favorable en el área foliar y en el número de hojas, brotes y racimos de plantas de uva de la selección clonal Riesling x Silvaner; en consecuencia, resultó ser el mejor tratamiento para la estimular la salida de reposo dela uva y una buena alternativa para reemplazar el uso de la cianamida hidrogenada en este cultivo. 


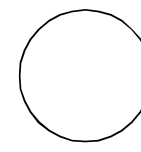

Botelho, R.; A. M aia; P. Paioli y M . M ontei ro. 2009. Efeito do extrato de alho na quebra de dormência de gemas de videiras e no controle in vitro do agente causal da antracnose (Elsinoe ampelina Shear). Rev. Bras. Viticult. 31(1), 10-16.

Botel ho, R.; A. Pavanello; E. Pires, M. Terra y M. M uller. 2007. Effects of chilling and garlic extract on bud dormancy release in Cabernet Sauvignon grapevine cuttings. Amer. J. Enol. Viticult. 58, 402-404.

Butrose, M. 1974. Climatic factors and fruitfulness in grapevines. A review. Hort. Abstr. 44, 319-326.

Disegna, E., A. Coniberti y E. Dellacassa. 2005. M edición de área foliar de la vid: una herramienta para producir vinos de calidad. Revista IN IA 4, 18-20.

Dokoozlian, N.K. 1999. Chilling temperature and duration interact on the budbreak of 'Perlette' grapevine cuttings. HortScience 34(6), 1054-1056.

EOT, Corrales. 2009. Alcaldía municipal del municipio de Corrales, Boyacá.

FAO. 2010. Faostat. Área cosechada, producción y rendimiento de uva. En: http: // faostat.fao.org/ site/ $567 /$ D esktopD efault . as px? Pagel $D=567 \#$ an cor; consulta: agosto de 2010.

Gómez, F. 2004. Zonificación. Terroir y la denominación de origen, en el fortalecimiento de los campesinos viticultores del valle del sol. Cultura Científica 2 , 15-25.

Hosoki, T.; H. H iura y M. H amada. 1985. Breaking bud dormancy in corms, tubers, and trees with sulfurcontaining compounds. H ortScience 20, 290-291.

Kubota N .; K. Toriu; Y. Yamane; T. Kawazu; T. H iguchi y S. Nishimura. 2003. Identification of active substances in chinese chive and rakkyo plants responsible for breaking bud dormancy in grape cuttings. J. Jap. Soc. Hort. Sci. 72(4), 268-274.

Kubota, N.; M. M atthew s; T. Takahagi y W. Kliewer. 2000. Effect of garlic preparations and of calcium and hydrogen cyanamides on budbreak of grapevines grown in greenhouses. Amer. J. Enol. Vitic. 51(4), 409-414.

Kubota, N.; M. M iyamuki; Y. Yamane; A. Kobayashi y F. M izutani. 1999. Breaking bud dormancy in

\section{REFERENCIAS BIBLIOGRÁFICAS}

grapevine cuttings with garlic volatiles. J. J ap. Soc. Hort. Sci. 68, 927-931.

Kubota, N .; G. Yanagisawa y K. Shimamura. 1987. Effect of root temperature on budbreak, shoot growth, and development of flower clusters of fruiting 'M uscat of Alexandria' vines under forced conditions. J. Jap. Soc. H ort. Sci. 56, 16-23.

Kubota, N. y M. Miyamuki. 1992. Breaking bud dormancy in grapevines with garlic paste. J. Amer. Soc. H ort. Sci. 117, 898-901.

M artínez, F. 1991. Biología de la vid. Fundamentos biológicos de la viticultura. Ed. Mundi-Prensa, M adrid

N avarrete, O. 2003. Deshidratación prematura en bayas de Vitis vinifera Cv. M erlot: efecto dela disminución del área foliar y aplicación de un antitranspirante. Trabajo de grado. Universidad de Talca, Chile.

Pérez, F. y W. Lira. 2005. Possible role of catalase in postdormancy bud break in grapevines. J. Plant Physiol. 162(3), 301-308.

Pinto, M .; W. Lira; H. U galde y F. Pérez. 2003. Fisiologia dela latencia delas yemas de vid: hipótesis actuales. Universidad de Chile. 16 p. En: http:// agronomia.uchile.cl/ extension / serviciosyproductos/gie/publicaciones; consulta: agosto de 2009.

Quijano, M . 2004. Ecología de una conexión solar. Dela adoración del sol al desarrollo vitivinícola regional. Cultura Científica 2, 5-9.

Quijano, M. 2001. Los vinos del valle del sol. Cultura Científica 1, 5-11.

Reynier, A. 1995. M anual de viticultura. 5a ed. Ed. $M$ undi-Prensa, $M$ adrid.

Salazar, D. y P. M elgarejo. 2005. Viticultura. Técnicas del cultivo de la vid, calidad de la uva y atributos de los vinos. Ed. M undi-Prensa, M adrid.

Settimi, L.; F. Davanzo; M. Faraoni; G. M icelli; D. Richmond y G. Calvert. 2005. Hidrogen cyanamide-related illnesses-I taly, 2002-2004. M orbidity and M ortality Weekly Report, Atlanta 54, 405-408. 
Toro, J. 1996. Como provocar una brotación uniforme. pp. 15-16. En: Galindo, J.; J. Toro y A. García (eds.). M anejo técnico del cultivo de la vid en el Valle del Cauca. Ceniuva, Colciencias, Bogotá.

Valor, O. y D. Bautista. 2001. Estudio fenológico de cuatro variedades de vid bajo las condiciones del tocuyo, estado de Lara. Bioagro 13(2), 57-63.

Valor, O. y D. Bautista. 1997. Brotación y fertilidad de yemas en tres cultivares de vid para vino. Agronomía Tropical 47(3), 347-358.

Vargas-Arispuro, I.; C. Corrales-M aldonado y $\mathrm{M}$. M artínez-T éllez. 2008. Compuestos derivados de ajo como agentes inductores de brotación en cultivo orgánico de uva de mesa. Chil. J. Agr. Res. 68(1), 94-101.

Vargas, G.; D. Bautista y P. Rabbion. 1994. Evaluación de variedades de vid para vino en condiciones tropicales. Agronomía Tropical 46(1), 18-29.

Wang, S.Y. y M . Faust. 1994. Changes in the antioxidant system associated with budbreak in 'Anna' apple ( $M$ alus domestica Borkh.) buds. J. Amer. Soc. Hort. Sci. 119, 735-741.

Westwood, M.N. 1993. Temperate-zone pomology. $3^{\text {rd }}$ ed. Timber Press, Portland, O R. 